\title{
AN EXTENSION OF DATA AUTOMATA THAT CAPTURES XPATH
}

\author{
MIKOŁAJ BOJAŃCZYK AND SŁAWOMIR LASOTA
}

Warsaw University

e-mail address: \{bojan,sl\}@mimuw.edu.pl

\begin{abstract}
We define a new kind of automata recognizing properties of data words or data trees and prove that the automata capture all queries definable in Regular XPath. We show that the automata-theoretic approach may be applied to answer decidability and expressibility questions for XPath.
\end{abstract}

\section{INTRODUCTION}

In this paper, we study data trees. In a data tree, each node carries a label from a finite alphabet and a data value from an infinite domain. We study properties of data trees, such as those defined in XPath, which refer to data values only by testing if two nodes carry the same data value. Therefore we define a data tree as a pair $(t, \sim)$ where $t$ is a tree over a finite alphabet and $\sim$ is an equivalence relation on nodes of $t$. Data values are identified with equivalence classes of $\sim$.

Recent years have seen a lot of interest in automata for data trees and the special case of data words. The general theme is that it is difficult to design an automaton which recognizes interesting properties and has decidable emptiness.

Decidable emptiness is important in XML static analysis. A typical question of static analysis is the implication problem: given two properties $\varphi_{1}, \varphi_{2}$ of XML documents (modeled as data trees), decide if every document satisfying $\varphi_{1}$ must also satisfy $\varphi_{2}$. Solving the implication problem boils down to deciding emptiness of $\varphi_{1} \wedge \neg \varphi_{2}$.

A common logic for expressing properties is XPath. For XPath, satisfiability is undecidable in general, even for data words, see 11. This means that most problems of static analysis are undecidable for XPath, e.g. the implication problem. Satisfiability is undecidable also for most other natural logics on data words or data trees, including first-order logic with predicates for order (or even just successor) and data equality.

The approach chosen in prior work was to find automata on data words or trees that would have decidable emptiness and recognize interesting, but necessarily weak, logics or

1998 ACM Subject Classification: F.1.1, H.2.3.

Key words and phrases: satisfiability of (regular) XPath queries, automata on data words and trees, data automata.

This work has been partially supported by the Polish government grants no. N206 008 32/0810 and N206 567840; and by the FET-Open grant agreement FOX, number FP7-ICT-233599.

LOGICAL METHODS IN COMPUTER SCIENCE
DOI:10.2168/LMCS-8 (1:05) 2012
(C) M. Bojańczyk and S. Lasota (c) Creative Commons 
fragments of XPath. These weak logics include: fragments of XPath without recursion or negation [1. 11]; first-order logic with two variables [4, 5]; forward-only fragments related to alternating automata $8-10,12$. The original automaton model for data words was [13. See [14] for a survey.

In this paper, we take a different approach. Any model that captures XPath will have undecidable emptiness. We are not discouraged by this, and try to capture XPath by something that feels like an "automaton". Three tangible goals are: 1. use the automaton to decide emptiness for interesting restrictions of data trees; 2 . use the automaton to prove easily that the automaton (and consequently XPath) cannot express a property; 3 . unify other automata models that have been suggested for data trees and words.

What is our new model? To explain it, we use logic. From a logical point of view, a nondeterministic automaton is a formula of the form $\exists X_{1} \ldots \exists X_{n} \varphi\left(X_{1}, \ldots, X_{n}\right)$, where the kernel $\varphi$ is relatively simple, e.g. it only talks about the relationship of labels in successive positions. As often in automata theory, when designing the automaton model, we try to use the prefix of existential set quantifiers as much as possible, in the interest of simplifying the kernel $\varphi$. For satisfiability, this is like a free lunch, since deciding satisfiability with or without the prefix are the same problem.

In the automaton model that we propose in this paper, the kernel $\varphi$ is of the form "for every class $X$ of $\sim$, property $\psi\left(X, X_{1}, \ldots, X_{n}\right)$ holds", where $\psi$ is an MSO formula that can use predicates for navigation (sibling order, descendant), predicates for testing labels from the finite alphabet, but not the predicate $\sim$ for data equality. The data $\sim$ is only used in saying that $X$ is a class. In the case of data words, this model is an extension of the data automata introduced in [5], which correspond to the special case when first-order quantifiers in $\psi$ range only over positions from $X$. For instance, our new model, but not data automata, can express the property "between every two different positions in the same class there is at most one position outside the class with label $a$ ".

The principal technical contribution of this paper is that the model above can recognize all unary queries of XPath. This proof is difficult, and takes over ten pages. We believe the real value of this paper lies in this proof, which demonstrates some powerful normalization techniques for formulas describing properties of data trees. Since the scope of applicability for these techniques will be clear only in the future; and since the appreciation of an "automaton model" may ultimately be a question of taste, we describe in more details the three tangible goals mentioned above.

1. The ultimate goal of this research is to find interesting classes of data trees which yield decidable emptiness for XPath. As a proof of concept, we define a simple subclass of data trees, called bipartite data trees, and prove that emptiness of our automata (and consequently of XPath) is decidable for bipartite data trees. This is only a preliminary result, we intend to find new subclasses in the future.

2. We use the automaton to prove that XPath cannot define certain properties. Proving inexpressibility results for XPath is difficult, because the truth value of an XPath query in a position $x$ might depend on the truth value of a subquery in a position $y<x$, which in turn might depend on the truth value of a subquery in a position $z>y$, and so on. On the other hand, our automaton works in one direction, so it is easier to understand its limitations. We use (an extension of) our automata to prove that for documents with two equivalence relations $\sim_{1}$ and $\sim_{2}$, some properties of two-variable first-order logic cannot be captured by XPath, which was an open question. 
3. We use the automaton to classify existing models for data words in a single framework. A problem with the research on data words and data trees is that the models are often incomparable in expressive power. In an upcoming paper ${ }^{1}$, we will show that many existing models can be seen as syntactic fragments of our automaton. We hope that this classification will underline more clearly what the differences are between the models.

\section{Preliminaries}

Trees. Trees are unranked, finite, and labeled by a finite alphabet $\Sigma$. We use the terms child, parent, sibling, descendant, ancestor, node in the usual way. The siblings are ordered. We write $x \leq y$ when $x$ is an ancestor of $y$. Every nonempty set of nodes $x_{1}, \ldots, x_{n}$ in a tree has a greatest common ancestor (the greatest lower bound wrt. $\leq$ ), which is denoted $\operatorname{gca}\left(x_{1}, \ldots, x_{n}\right)$.

Let $t$ and $s$ be two trees, over alphabets $\Sigma$ and $\Gamma$, respectively, that have the same sets of nodes. We write $t \otimes s$ for the tree over the product alphabet $\Sigma \times \Gamma$ that has the same nodes as $s$ and $t$, and where every node has the label from $t$ on the first coordinate, and the label from $s$ on the second coordinate. If $X$ is a set of nodes in a tree $t$, we write $t \otimes X$ for the tree $t \otimes s$, where $s$ is the tree over alphabet $\{0,1\}$, whose nodes are the nodes of $t$ and whose labeling is the characteristic function of $X$.

Regular tree languages and transducers. We use the standard notion of regular tree languages for unranked trees [7]. We also use transductions, which map trees to trees. Let $\Sigma$ be an input alphabet and $\Gamma$ an output alphabet. A regular tree language $f$ over the product alphabet $\Sigma \times \Gamma$ can be interpreted as a binary relation, which contains pairs $(s, t)$ such that $s \otimes t \in f$. We use the name letter-to-letter transducer for such a relation, underlining that the trees in a pair $(s, t) \in f$ must have the same nodes. In short, we simply say transducer. Observe that the transducer is nondeterministic. We often treat a transducer as a function that maps an input tree to a set of output trees, writing $t \in f(s)$ instead of $(s, t) \in f$.

Data trees. A data tree is a tree $t$ equipped with an equivalence relation $\sim$ on its nodes that represents data equality. We use the name class for equivalence classes of $\sim$.

Queries. Fix an input alphabet. We use the name $n$-ary query for a function $\phi$ that maps a tree $t$ over the input alphabet to a set $\phi(t)$ of $n$-tuples of its nodes. In this paper, we deal with queries of arities $0,1,2$ and 3 , which are called boolean, unary, binary and ternary. We also study queries that input a data tree $(t, \sim)$; they output a set of node tuples $\phi(t, \sim)$ as well.

\footnotetext{
${ }^{1}$ This paper is a journal version of a LICS 2010 paper 3, which included a rough description of the classification mentioned in item 3. However, a thorough explanation of the classification requires much space, and uses different techniques than the results in this paper. Therefore, we plan to present the classification in a separate paper.
} 
MSO. Logic is a convenient way of specifying queries, both for trees and data trees. We use monadic second-order logic (MSO). In a given tree, or a data tree, a formula of MSO is allowed to quantify over nodes of the tree using individual variables $x, y, z$, and also over sets of nodes using set variables $X, Y, Z$. A formula $\phi$ with free individual variables $x_{1}, \ldots, x_{n}$ defines an $n$-ary query, which selects in a tree $t$ the set $\phi(t)$ of tuples $\left(x_{1}, \ldots, x_{n}\right)$ that make the formula true. To avoid confusion, we use round parentheses for the tree input of a query, $\phi(t)$, and square parentheses for indicating the free variables of a query. The two parenthesis can appear together, e.g. $\phi\left[x_{1}, \ldots, x_{n}\right](t)$ will be the set of $n$-tuples selected in a tree $t$ by a query with free variables $x_{1}, \ldots, x_{n}$.

When working over trees without data, MSO formulas use binary predicates for the child and next-sibling relations (that allow to define descendant and following-sibling relations), as well as a unary predicate for each label. Queries defined by MSO with these predicates are called regular queries (of course, regular queries can also be characterized in terms of automata). When working over data trees, we additionally allow a binary predicate $\sim$ to test data equality. A query using $\sim$ is no longer called regular. For instance, the following unary query selects positions that have classes of size at least two:

$$
\varphi(x) \quad=\quad \exists y \quad x \neq y \wedge x \sim y .
$$

Extended Regular XPath. We define a variant of XPath that works over data trees. For unary queries, the variant is an extension of XPath, thanks to including MSO as part of its syntax. We call the variant Extended Regular XPath. Unlike XPath, Extended Regular XPath allows for queries of arbitrary arity. Expressions of Extended Regular XPath are defined below.

- Let $\Gamma=\left\{\phi_{1}, \ldots, \phi_{n}\right\}$ be a set of already defined unary queries of Extended Regular XPath, which will be treated as unary predicates. In the induction base, the set $\Gamma$ is necessarily empty. Suppose that $\varphi\left[x_{1}, \ldots, x_{m}\right]$ is an MSO query that uses unary predicates for queries from $\Gamma$, unary predicates for letters of the input alphabet, and the binary child and nextsibling predicates. Then $\varphi$ is an $m$-ary query of Extended Regular XPath. It is important that $\varphi$ does not introduce any new use of the data equality predicate $\sim$, all appearances of $\sim$ are reserved to the queries from $\Gamma$.

- Suppose that $\varphi\left[x, y_{1}, y_{2}\right]$ is a ternary query of Extended Regular XPath. Then the following property of $x$ is a unary query of Extended Regular XPath

$$
\exists y_{1} \exists y_{2} \quad y_{1} \sim y_{2} \wedge \varphi\left[x, y_{1}, y_{2}\right] .
$$

Likewise for $y_{1} \nsim y_{2}$ instead of $y_{1} \sim y_{2}$.

The definition above allows for queries of any arity. In the paper, we will be principally interested in queries of arity one, and the queries of arity at most three used to build them. By abuse of nomenclature, we will write XPath instead of Extended Regular XPath.

Binary trees. A binary tree is a tree where each node has at most two children. Although the interest of XPath is mainly for unranked trees, we assume in the proofs that trees are binary. This assumption can be made because XPath, as well as the models of automata introduced later on, are stable under the usual first-child / next-sibling encoding in the following sense. A language $L$ of unranked data trees can be expressed by a boolean XPath query if and only if the set of binary encodings of trees from $L$ can be expressed by a boolean XPath query. A similar, though more technical, statement holds for unary queries. 
Words will be considered as a special case of binary trees where each node has at most one child.

\section{Class automata}

In this section we define a new type of automaton for data trees, called a class automaton, and state the main result: class automata capture all queries definable in XPath.

A class automaton is a type of automaton that recognizes properties of data trees. A class automaton is given by: an input alphabet $\Sigma$, a work alphabet $\Gamma$, a nondeterministic letter-to-letter tree transducer $f$ from the input alphabet $\Sigma$ to the work alphabet $\Gamma$, and a regular tree language on alphabet $\Gamma \times\{0,1\}$, called the class condition. The class automaton accepts a data tree $(t, \sim)$ over input alphabet $\Sigma$ if there is some output $s \in f(t)$ such that for every class $X$, the class condition contains the tree $s \otimes X$.

Example 1. Consider an input alphabet $\Sigma=\{a, b\}$. Let $L$ be the data trees where some class contains at least three nodes with label $a$. This language is recognized by a class automaton. The work alphabet is $\Gamma=\{a, c\}$. The transducer guesses three nodes with label $a$, and outputs $a$ on them, other nodes get $c$. The class condition consists of trees $s \otimes X$ over alphabet $\Gamma \times\{0,1\}$ where $X$ contains all or none of the nodes with label $a$. Note that the class condition does not inspect positions outside $X$.

Example 2. Let $K$ be the set of data words over $\Sigma=\{a, b\}$ where each class has exactly two positions $x<y$, and there is at most one $a$ in the positions $\{x+1, \ldots, y-1\}$. In the class automaton recognizing $K$, the transducer is the identity function, and the class condition is

$$
\Sigma_{0}^{*} \cdot \Sigma_{1} \cdot b_{0}^{*} \cdot\left(a_{0}+\epsilon\right) \cdot b_{0}^{*} \cdot \Sigma_{1} \cdot \Sigma_{0}^{*}
$$

where $\Sigma_{i}$ is a shortcut for $\Sigma \times\{i\}$, likewise for $a_{i}$ and $b_{i}$.

Comparison to data automata. Class automata are closely related to data automata introduced in [5]. Data automata were defined for data words. Since it is not clear what the correct tree version thereof is, we just present the version for data words. Like a class automaton, a data automaton has an input alphabet $\Sigma$, a work alphabet $\Gamma$, and a nondeterministic letter-to-letter transducer $f$ (this time only for words). The difference is in the class condition, which is less powerful in a data automaton. In a data automaton, the class condition is a word language over $\Gamma$, and not $\Gamma \times\{0,1\}$. The data automaton accepts a data word $(w, \sim)$ if there is some output $v \in f(w)$ such that for every class $X$, the class condition contains the subsequence of $v$ obtained by only keeping positions from $X$. In the realm of data words, data automata can be seen as a special case of class automata, where the class condition is only allowed to look at positions from the current class. The language $L$ in Example 1 can be recognized by a data automaton (in the case of words), while the language $K$ in Example 2 is a language that can be recognized by class automata, but not data automata.

The difference between data automata and class automata is crucial for decidability of emptiness. Data automata have decidable emptiness [5], the proof being a reduction to reachability in Vector Addition Systems with States. Class automata have undecidable emptiness, because they capture the logic XPath, which has undecidable satisfiability. Also, 
a direct and simple proof of undecidable emptiness for class automata can be given, by encoding runs of two-counter machines, without going through the difficult reduction from XPath.

Closure properties. Suppose that $f: \Sigma_{1} \rightarrow \Sigma_{2}$ is any function. We extend $f$ to a function $\hat{f}$ from data trees over alphabet $\Sigma_{1}$ to data trees over alphabet $\Sigma_{2}$, by just changing the labels of nodes, and not the tree structure or data values. We use the name relabeling for any such function $\hat{f}$.

Lemma 1. Languages of data trees recognized by class automata are closed under union, intersection, images under relabelings, and inverse images under relabelings.

Proof. The inverse images are the simplest: the letter-to-letter tree transducer in the class automaton is composed with the relabeling. For intersection, one uses Cartesian product. For union and images under relabelings, one uses nondeterminism.

Evaluation. The evaluation problem (given an automaton and a data word/tree, check if the latter is accepted by the former) is NP-complete, even for a fixed data automaton (cf. [2]). Hence it is also NP-complete for class automata, which extend data automata.

Class automata as a fragment of MSO. As mentioned in the introduction, one can see a class automaton as a restricted type of formula of monadic second-order logic. This is a formula of the form:

$$
\exists X_{1} \cdots \exists X_{n} \forall X \operatorname{class}(X) \Rightarrow \varphi\left(X_{1}, \ldots, X_{n}, X\right)
$$

where $X_{1}, \ldots, X_{n}, X$ are variables for sets of nodes, the class formula is

$$
\operatorname{class}(X)=\exists y \forall x \quad x \in X \Longleftrightarrow y \sim x
$$

and $\varphi$ is a formula of MSO that does not use $\sim$. Formulas of the above form recognize exactly the same languages of data trees as class automata. For translating a class automaton to a formula, one uses the variables $X_{1}, \ldots, X_{n}$ to encode the output of the transducer, and the formula $\varphi$ to test two things: a) the variables $X_{1}, \ldots, X_{n}$ encode a legitimate output of the transducer; and b) the class condition holds for $X$.

Main result. The main result of this paper is Theorem 1 below, which says that unary XPath queries over data trees can be recognized by class automata. To state the theorem, we need to say how a class automaton recognizes a unary query. We do this by encoding a unary query $\phi$ over data trees as a language of data trees:

$$
L_{\phi}=\{(t \otimes X, \sim):(t, \sim) \text { is a data tree, } X=\phi(t, \sim)\} .
$$

In other words, the language consists of data trees decorated with the set of nodes selected by the query. This encoding does not generalize to binary queries.

Theorem 1. Every unary XPath query over data trees can be recognized by a class automaton.

We begin the proof of Theorem 1, mainly to show where the difficulties appear. Then, we lay out the proof strategy in more detail. When referring to the language of a unary query, we mean the encoding above. 
We do an induction on the size of the unary query. The base case, when the query is a label $a$, is straightforward. Consider now the induction step, with a unary query

$$
\phi[x]=\exists y_{1} \exists y_{2} \quad y_{1} \sim y_{2} \wedge \varphi\left[x, y_{1}, y_{2}\right]
$$

as in (2.1). (The same argument works for the case where $y_{1} \not y_{2}$.) Let $\phi_{1}, \ldots, \phi_{n}$ be all the unary XPath subqueries that appear in $\varphi$. By the induction assumption, the languages of the subqueries are recognized by class automata $\mathcal{A}_{1}, \ldots, \mathcal{A}_{n}$. Let the variables $X, X_{1}, \ldots, X_{n}$ denote sets of nodes. Consider the set $L$ of data trees

$$
\left(t \otimes X \otimes X_{1} \otimes \cdots \otimes X_{n}, \sim\right)
$$

such that a) for each $i \in\{1, \ldots, n\}$, the data tree $\left(t \otimes X_{i}, \sim\right)$ is accepted by the automaton $\mathcal{A}_{i}$; and b) $X$ is the set of nodes selected by the query $\phi^{\prime}$ obtained from $\phi$ by replacing each subquery $\phi_{i}$ with "has 1 on coordinate corresponding to $X_{i}$ ". Suppose that the language of $\phi^{\prime}$ is recognized by a class automaton. Then so is $L$, by closure of class automata under intersection and inverse images of projections, see Lemma 1. Finally, the language of $\phi$ is the image of $L$ under the projection which removes the labels describing the sets $X_{1}, \ldots, X_{n}$.

It remains to show that $\phi^{\prime}$ is recognized by a class automaton (the advantage of $\phi^{\prime}$ over $\phi$ is that it uses data equality $\sim$ only once, to say that $\left.y_{1} \sim y_{2}\right)$. A major part of this paper is devoted to this case, which is stated in the following proposition.

Proposition 1. Class automata can recognize queries

$$
\phi[x]=\exists y_{1} \exists y_{2} \quad y_{1} \sim y_{2} \wedge \varphi\left[x, y_{1}, y_{2}\right],
$$

where $\varphi$ is a regular ternary query (i.e. $\varphi$ does not use $\sim$ ). Likewise for $y_{1} \nsim y_{2}$.

Proof strategy. The construction of the automaton for $\phi[x]$ is spread across several sections. In Section 3.2, we introduce the main concepts underlying the proof. In particular, we define a new complexity measure for binary relations on tree domains, called guidance width, that seems to be of independent interest. In Section 3.3 we start the proof itself, formulate an induction, and reduce Proposition 1 to a more technical Theorem 2, Then in Section 4 we identify a simplified form of queries appearing in Theorem 2 and show how arbitrary queries can be transformed to the simplified form. Finally, Section 5 contains the proof of Theorem 2 for these simplified queries, the heart of the whole proof.

3.1. Discussion of the proof. In this section, we discuss informally the concepts that appear in the proof of Theorem 11. For the purpose of illustration, we use words.

We begin our discussion with words without data. For a regular binary query $\varphi[x, y]$, consider the unary query

$$
\psi[x]=\exists y \varphi[x, y] .
$$

We use the name witness function in a word $w$ for a function which maps every position $x$ satisfying $\psi$ to some $y$ such that $\varphi[x, y]$ holds. Consider, as an example, the case where $\varphi[x, y]$ says that there exists exactly one $z$ that has label $a$ and satisfies $x<z<y$. The following picture shows a witness function.

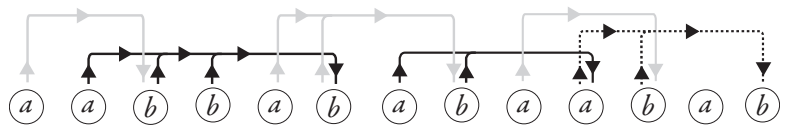


The way the picture is drawn is important. The witness function is recovered by following arrowed lines. The arrowed lines are colored black, dashed black, or gray, in such a way that no position is traversed by two arrowed lines of the same color. With the formula $\psi$ in the example, any input word has a witness function that can be drawn with three colors of arrowed lines. This can be generalized to arbitrary MSO binary queries; the number of colors depends only on the query, and not the input word.

The above observation may be used to design a nondeterministic automaton recognizing a property like $\forall x \psi[x]$. The automaton would guess the labeling by arrows and then verify its correctness. The number of states in the automaton would grow with the number of colors; hence the need for a bound on the number of colors. Of course, there are other ways of recognizing $\forall x \psi[x]$, but we talk about the coloring since this is the technique that will work with data.

We now move to data words. Consider a unary query

$$
\psi[x]=\exists y_{1} \exists y_{2} \quad y_{1} \sim y_{2} \wedge \varphi\left[x, y_{1}, y_{2}\right],
$$

where $\varphi\left[x, y_{1}, y_{2}\right]$ says that $y_{1}<x \leq y_{2}$, there is exactly one $a$ label in the positions $\left\{y_{1}, \ldots, x-1\right\}$ and there is exactly one $b$ label in the positions $\left\{x, \ldots, y_{2}\right\}$. The query $\psi[x]$ is an example of a query as in Proposition 1. Consider the following data word (the labels are blank, $a$ and $b$, the data values are $1 \ldots 6$ ).

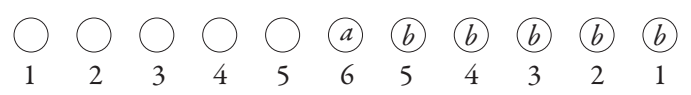

Say $x$ is the first node with label $b$. This node is selected by $\psi$. Consider the pairs $\left(y_{1}, y_{2}\right)$ required by $\psi[x]$, which we call witnesses. The only possibility for $y_{2}$ is $x$ itself; thus $y_{1}$ is also determined, as the only other position with the same data value. So there is only one witness pair. The same situation holds for all other positions with label $b$, which are the only positions selected by $\psi$. The drawing below shows how witness pairs are assigned to positions.

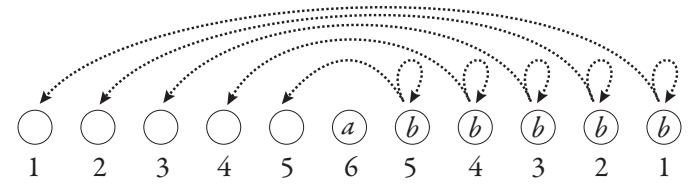

We would like to draw this picture with colored arrows, as we did for the first example of witness functions. If we insist on drawing arrows that connect each position $x$ with its corresponding witness $y_{1}$, then we will need 5 colors as the middle position (labeled by a) is traversed by 5 arrows; the picture also generalizes to any number of colors. On the other hand, connecting each position $x$ with its corresponding $y_{2}$ (a self-loop) requires only one color. We can symmetrically come up with instances of data words where connecting each node $x$ to $y_{2}$ requires an unbounded number of colors.

A consequence of our main technical result, Theorem 2, is that a bounded number of colors is sufficient if we want to perform the following task: for each position $x$ selected by $\psi$, choose some witness pair $y_{1} \sim y_{2}$, and connect $x$ to either $y_{1}$ or $y_{2}$. The bound depends only on $\psi$; in particular, the bound does not depend on input data tree.

The concepts of witness functions and coloring are defined more precisely below. 
3.2. The core result. We will state some technical results for a structure more general than a data tree, namely a graph tree. A graph tree is a tree $t$ endowed with an arbitrary symmetric binary relation $E$ over its nodes. A data tree is the special case of a graph tree where $E$ is an equivalence relation.

Witness functions. Let $\varphi\left[x, y_{1}, y_{2}\right]$ be a regular query (think of Proposition 1), and consider a graph tree $(t, E)$. We are interested in triples $\left(x, y_{1}, y_{2}\right)$ selected by $\varphi$ in $t$ such that $\left(y_{1}, y_{2}\right) \in E$. (Think of $E$ being either the data equivalence relation $\sim$, or its complement.) Consider any such triple. The node $x$ is called the source node; the notion of source node is relative to the query $\varphi$ and relation $E$, which will usually be clear from the context and not mentioned explicitly. The pair $\left(y_{1}, y_{2}\right)$ is called the witness pair, $y_{1}$ is called the first witness, and $y_{2}$ is called the second witness. These notions are all relative to a given $x$, but if we do not mention the $x$, then $x$ is quantified existentially. Let $X$ be a set of nodes in a graph tree (not necessarily containing all nodes). A witness function for $\varphi$ and $X$ in a graph tree is a function which maps every node $x \in X$, treated as a source node, to some (first or second) witness. There may be many witness functions, since for each node we can choose to use either a first witness or a second witness, and there may be multiple witness pairs.

The key technical result of this paper is that one can always find a witness function of low complexity. The notion of complexity is introduced below.

Guidance width. A guide in a tree $t$ is given by two nonempty sets of source nodes and target nodes. The support of the guide is the set of all nodes and edges on (the shortest) paths that connect some source node with a target node, including all the source and target nodes. A guide conflicts with another guide if their supports intersect. We write $\pi$ for guides.

A guidance system is a set of guides $\Pi$. It induces a relation containing all pairs $(x, y)$ of tree nodes such that $x$ is a source and $y$ a target in some guide in $\Pi$. An $n$-color guidance system is a guidance system whose guides can be colored by $n$ colors so that conflicting guides have different colors. The guidance width of a binary relation $R$ on tree nodes is the smallest $n$ such that some $n$-color guidance system induces $R$.

In the proof we will only consider guidance systems for relations $R$ that are partial functions from tree nodes to tree nodes. In such cases, it is sufficient to restrict to deterministic guides, i.e., those with precisely one target node. From now on, if not stated otherwise, a guidance system will be implicitly assumed to contain only deterministic guides.

Witness functions of bounded width. We are now ready to state the main technical result, which forms the core of the proof of Theorem 1 .

Theorem 2. Let $\varphi$ be a regular ternary query. There exists a constant $m$, depending only on $\varphi$, such that in every graph tree, every set of source nodes has some witness function of guidance width at most $m$.

In other words, regular ternary queries have witness functions of bounded guidance width. Before proving the theorem, we show how it implies Theorem 1 . 
3.3. From Theorem 2 to Proposition 1. We show how Theorem 2 implies the last remaining piece of Theorem 1, namely Proposition 1. Consider a unary query $\phi[x]$ as in the statement of Proposition 1. We begin with the case when $\phi[x]$ is of the form

$$
\phi[x]=\exists y_{1} \exists y_{2} \quad y_{1} \sim y_{2} \wedge \varphi\left[x, y_{1}, y_{2}\right] .
$$

We need to find a class automaton that accepts the data trees $(t \otimes X, \sim)$ where $X$ is the set of all nodes selected by $\phi$ in the data tree $(t, \sim)$. The class automaton will test the conjunction of two properties:

Completeness. Each node selected by $\phi$ in $(t, \sim)$ is in $X$.

Correctness. Each node in $X$ is selected by $\phi$ in $(t, \sim)$.

Recall that $\varphi$ is a regular query. We give separate class automata for the two properties. Completeness is simple. It can be rephrased as for every class $Y$ and triple $\left(x, y_{1}, y_{2}\right)$ selected by $\varphi$, if $y_{1}, y_{2} \in Y$ then $x \in X$.

This is the type of property class automata are designed for: for every class, test a regular property. (Recall the discussion on class automata as a fragment of MSO.) Correctness is the difficult property, since the order of quantifiers is not the same as in a class automaton:

for every $x \in X$ there is a class $Y$ and $y_{1}, y_{2} \in Y$ such that $\left(x, y_{1}, y_{2}\right)$ is selected by $\varphi$.

Our solution is to use, as a part of the class automaton to be designed, a guidance system given by Theorem 2 .

Apply Theorem 2 to $\varphi$, yielding a constant $m$. The class automaton for the correctness property works as follows. Given an input data tree $(t \otimes X, \sim)$, it guesses an $m$-color guidance system; let $R$ stand for the induced relation. The automaton then checks the two conditions below.

A. For every $x \in X$ there is some $y$ with $x R y$.

B. For every class $Y$, if $x R y, x \in X, y \in Y$, then either $\left(x, y, y^{\prime}\right)$ or $\left(x, y^{\prime}, y\right)$ is in $\varphi(t)$, for some $y^{\prime} \in Y$.

If the class automaton accepts, then clearly every position in $X$ is a source node. Conversely, if all nodes in $X$ are source nodes, then there is an accepting run of the above class automaton. This accepting run uses the guidance system for the witness function from Theorem 2,

This completes the proof for the case when $\phi[x]$ requires $y_{1} \sim y_{2}$. For the case $y_{1} \nsim y_{2}$, the proof is almost the same, except for two changes. The first change is that we apply Theorem 2 to the graph trees $(t, E)$, obtained from data trees $(t, \sim)$ by taking as $E$ the complement of $\sim$. This explains why Theorem 2 is formulated for graph trees and not just data trees. The second change is that we write $y^{\prime} \notin Y$ instead of $y^{\prime} \in Y$ at the end of condition B.

\section{Simplifying THE QUERY}

Before proving Theorem 2 , we formulate two simplifying conditions about the regular query $\varphi\left[x, y_{1}, y_{2}\right]$.

For two nodes $x, y$ in a tree $t$, we write $\operatorname{word}_{t}(x, y)$ for the sequence of labels on the unique shortest path from $x$ to $y$ in $t$, including $x$ and $y$. We omit the subscript $t$ when a 
tree is clear from the context. Note that $\operatorname{word}_{t}(x, y)$ is always nonempty and $\operatorname{word}_{t}(x, x)$ is the label of $x$.

The two conditions about the query $\varphi\left[x, y_{1}, y_{2}\right]$ are:

(1) All selected triples satisfy $y_{1}<x<y_{2}$.

(2) Whether or not a triple is selected depends only on the words $\operatorname{word}_{t}\left(y_{1}, x\right)$ and word $_{t}\left(x, y_{2}\right)$. It does not depend on nodes outside the path from $y_{1}$ to $y_{2}$.

The goal of this section is to reduce Theorem 2 to the case when $\varphi\left[x, y_{1}, y_{2}\right]$ is a simplified query, as defined above. This simplification is achieved in several steps. (In the case of words, the simplification would be standard, but for trees it requires new ideas about guidance systems.) Formally, in this section we show that Theorem 2 follows from Theorem 3 (deliberately formulated as late as in the forthcoming Subsection 4.7) that only speaks about simplified queries. Theorem 3 itself is proved in Section 5.

4.1. Generalized witness functions. Fix any number $n \in \mathbb{N}$, although we will be mainly interested in $n \in\{1,2\}$. Consider a regular query $\varphi\left[x, y_{1}, \ldots, y_{n}\right]$ over trees. Consider now a tree $t$ together with a set $E$ of $n$-tuples of nodes in $t$. As before, the idea is that $E$ gives a constraint on the witness variables. A witness tuple for a node $x$ is a tuple $\left(y_{1}, \ldots, y_{n}\right) \in E$ such that $\left(x, y_{1}, \ldots, y_{n}\right)$ is selected by $\varphi$ in $t$. In this case, we say that $x$ is a source, and $y_{i}$ is an $i$-th witness for $x$ (the other variables are quantified existentially).

A witness function for $\varphi$ and a set of source nodes $X$ in $(t, E)$ is a function which assigns to each node $x \in X$ some witness (an $i$-th witness for some $i$, with $i$ depending on $x)$.

We say that a regular query $\varphi\left[x, y_{1}, \ldots, y_{n}\right]$ has witness functions of guidance width $m$ if for every tree $t$, every choice $E$ of $n$-tuples of nodes of $t$ and every set $X$ of source nodes in $(t, E)$, there is a witness function for $\varphi$ and $X$ of guidance width at most $m$. A query $\varphi$ has witness functions of bounded guidance width if some such $m$ exists.

4.2. Three arrangements. By an arrangement of the nodes $x, y_{1}, y_{2}$ in a tree we mean the information on how these nodes, and their greatest common ancestors

$$
\operatorname{gca}\left(x, y_{1}\right) \quad \operatorname{gca}\left(x, y_{2}\right) \quad \operatorname{gca}\left(y_{1}, y_{2}\right)
$$

are related with respect to the descendant ordering. We distinguish three different arrangements, pictured below.
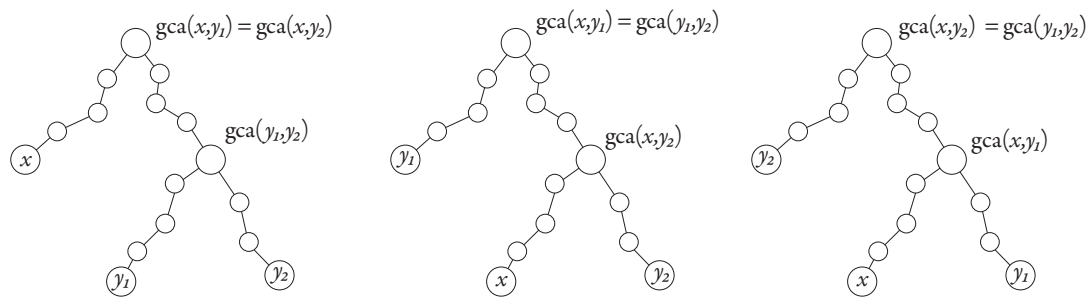

These arrangements correspond, respectively, to the following situations.

$$
\begin{aligned}
& \operatorname{gca}\left(x, y_{1}\right)=\operatorname{gca}\left(x, y_{2}\right) \leq \operatorname{gca}\left(y_{1}, y_{2}\right) \\
& \operatorname{gca}\left(x, y_{1}\right)=\operatorname{gca}\left(y_{1}, y_{2}\right) \leq \operatorname{gca}\left(x, y_{2}\right) \\
& \operatorname{gca}\left(x, y_{2}\right)=\operatorname{gca}\left(y_{1}, y_{2}\right) \leq \operatorname{gca}\left(x, y_{1}\right)
\end{aligned}
$$


The arrangements are not contradictory, for instance the case $x=y_{1}=y_{2}$ is covered by all three. The slightly more general case $y_{1}=y_{2}$ that essentially represents binary queries $\varphi[x, y]$, is fully covered by A1.

Lemma 2. We may assume without loss of generality that all the triples selected by $\varphi$, as in the statement of Theorem 2 , have the same arrangement.

Proof. Otherwise we can split $\varphi$ into a union of three queries, one for each arrangement, and then combine the three separate guidance systems.

4.3. Path-based queries. Let us fix one of the arrangements. There are four words $w_{1}, w_{2}, w_{3}, w_{4}$ that will interest us. These are shown on the picture below for the arrangement (A1) only, but the reader can easily see the situation for all other arrangements.
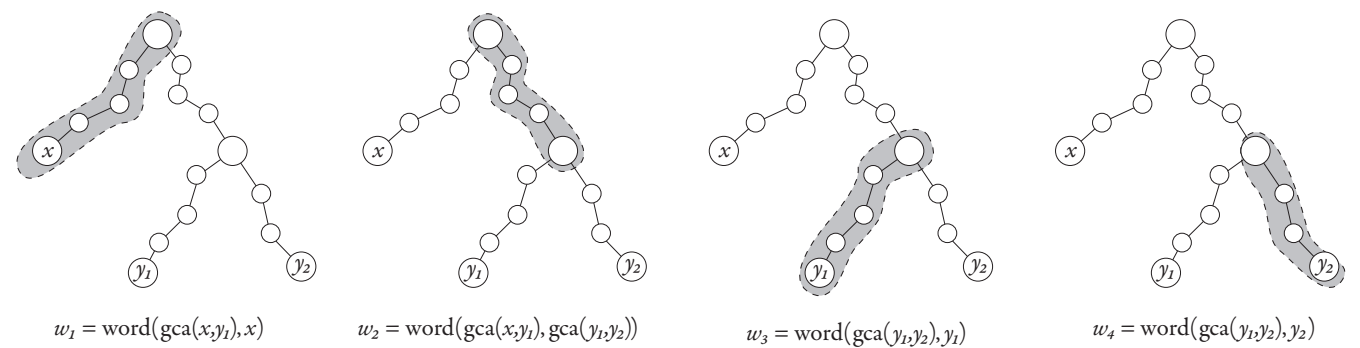

$w_{2}=\operatorname{word}\left(\operatorname{gca}\left(x, y_{1}\right), \operatorname{gca}\left(y_{1}, y_{2}\right)\right)$

$w_{3}=\operatorname{word}\left(\operatorname{gca}\left(y_{1}, y_{2}\right), y_{1}\right)$

$w_{4}=\operatorname{word}\left(\operatorname{gca}\left(y_{1}, y_{2}\right), y_{2}\right)$

A regular query $\varphi\left[x, y_{1}, y_{2}\right]$ is called path-based if its truth value depends only on some regular properties of the four words $w_{1}, \ldots, w_{4}$. The precise definition of path-based queries we use in this paper is in terms of monoids. A query that selects triples only in arrangement (A1) is called path-based if there exists a monoid morphism

$$
\alpha: \Sigma^{*} \rightarrow S
$$

such that membership $\left(x, y_{1}, y_{2}\right) \in \varphi(t)$ depends only on the values assigned by $\alpha$ to the words $w_{1}, \ldots, w_{4}$. In other words, there is a set of accepting quadruples $F \subseteq S^{4}$ such that $\left(x, y_{1}, y_{2}\right)$ belongs to $\varphi(t)$ if and only if

$$
\left(\alpha\left(w_{1}\right), \ldots, \alpha\left(w_{4}\right)\right) \in F .
$$

An analogous definition of path-based queries is given for the other arrangements A2 and $\mathrm{A} 3 \mathrm{~B}$.

Lemma 3. We may assume without loss of generality that $\varphi$, as in the statement of Theorem 2, is path-based.

Proof. The key observation is that there is a functional letter-to-letter transducer $f$ and a path-based query $\gamma$ such that $\varphi=\gamma \circ f$, i.e. for a tree $t$ the set $\varphi(t)$ of tuples selected by $\varphi$ in $t$ is the same as the set of tuples selected by $\gamma$ in $f(t)$. The observation can be proved using logical methods (the transducer computes MSO theories) or using automata methods (the transducer computes state transformations).

To prove the lemma, we need to show that if Theorem 2 is true for the path-based queries $\gamma$, then it is also true for arbitrary ternary queries $\varphi$. But this is straightforward, as $\varphi$ and $\gamma$ have the same witness functions in trees $t$ and $f(t)$, respectively. 
4.4. Composing guidance systems. In the sequel we will compose guidance systems as outlined in the lemma below. For two partial functions $f, g$ on the set of nodes of a tree, by the composition $g \circ f$ we mean, somewhat non-standardly, the partial function with the same domain as $f$, and defined as follows:

$$
(g \circ f)(x)= \begin{cases}g(f(x)) & \text { if } \mathrm{g} \text { is defined on } f(x) \\ f(x) & \text { otherwise. }\end{cases}
$$

Lemma 4. Let $f, g$ be partial functions on the set of nodes of a tree, of guidance width $m_{1}$ and $m_{2}$, respectively. Then their composition $g \circ f$ is of guidance width at most $2 m_{1} m_{2}$.

Proof. Fix a tree $t$ together with some $m_{1^{-}}$and $m_{2}$-color guidance systems $\Pi_{f}$ and $\Pi_{g}$, inducing $f$ and $g$, respectively. We will show existence of a $2 m_{1} m_{2}$-color guidance system for $g \circ f$.

As the first step, combine $\Pi_{f}$ and $\Pi_{g}$ as follows: a node $x$ is first guided by $\Pi_{f}$, and then, if $g$ is defined on $f(x)$, guided by $\Pi_{g}$ to its final destination. Formally, $\Pi$ contains those guides of $\Pi_{f}$ whose destination node is not in the domain of $g$; and moreover a number of guides that are composed of at least two guides, to be described now.

Fix a pair of colors $(k, l)$, where $k$ is a color used in $\Pi_{f}$ and $l$ is a color used in $\Pi_{g}$. A composed guide, colored by the pair $(k, l)$, is derived from one $l$-colored guide from $\Pi_{g}$, say $\pi$, and all those $k$-colored guides from $\Pi_{f}$ whose destination node is a source node of $\pi$, The source nodes of the composed guide are all source nodes of all the above-mentioned $k$-colored guides from $\Pi_{f}$. The target node of the composed guide is the target node of $\pi$.

We will focus on the composed guides only. (A 'non-composed' guide in $\Pi$, say colored $k$, may be safely considered as colored by $(k, l)$, for any $l$.)

The above coloring, using $m_{1} m_{2}$ colors, is not satisfactory as same colored guides may be in conflict. We will show how to resolve these conflicts by introducing an additionally distinguishing piece of data into the colors. Fix a color pair $(k, l)$ as above. Note that a conflict may only arise when the $\Pi_{g}$-part $\left(l\right.$-colored in $\left.\Pi_{g}\right)$ of one $(k, l)$-colored guide, say $\pi_{1}$, conflicts with the $\Pi_{f}$-part ( $k$-colored in $\Pi_{f}$ ) of another same colored guide, say $\pi_{2}$. Consider an undirected graph $G$, whose nodes are all $(k, l)$-colored guides; there is an edge between $\pi_{1}$ and $\pi_{2}$ in the graph if the abovementioned conflict arises.

We claim that the graph $G$ is a forest, i.e., a disjoint union of trees. Towards a contradiction, suppose that $G$ has a cycle consisting of $n$ pairwise different guides $\pi_{1}, \ldots, \pi_{n}$. Take $\pi_{n+1}=\pi_{1}$. Let $x_{1}, \ldots, x_{n}$ denote arbitrarily chosen nodes witnessing the conflicts, i.e., $x_{i}$ belongs to the supports of guides $\pi_{i}$ and $\pi_{i+1}$. In $\pi_{i+1}$, for any $i \leq n$, there is a unique path from $x_{i}$ to $x_{i+1}$ (take $x_{n+1}$ as $x_{1}$ ), denote it $p_{i} ; p_{i}$ always uses a path of a guide from $\Pi_{f}$, colored $k$, and a path of a guide from $\Pi_{g}$, colored $l$. As the $k$-colored guides never conflict, and likewise the $l$-colored ones, the $k$-colored part of $p_{i}$ is separated from the same colored part of $p_{i+1}$ by at least one $l$-colored edge; thus the paths $p_{i}$ are nonempty, i.e., $x_{i} \neq x_{i+1}$. Assume that $x_{1}, \ldots, x_{n}$ are pairwise distinct (if this is not the case, i.e., $x_{i}=x_{j}$, consider $x_{i}, \ldots, x_{j-1}$ instead; and consider $\pi_{i}, \ldots, \pi_{j-1}$ instead of $\left.\pi_{1}, \ldots, \pi_{n}\right)$.

Now we are prepared to obtain a contradiction, thus proving that $G$ is a forest. If two paths $p_{i}$ and $p_{i+1}$ share an edge adjacent to $x_{i+1}$, the edge may be removed from both paths; this clearly forces $x_{i+1}$ to be replaced appropriately. Thus the paths can be made edge-disjoint; moreover we keep the $x_{i}$ nodes pairwise distinct, argued as above. Hence the paths $p_{1}, \ldots, p_{n}$ form a cycle in the tree $t$, a contradiction. 
Knowing that $G$ is a forest, we may easily label its nodes by two numbers 1,2 , level by level, starting from an arbitrary leaf in any connected component. This additional numbering, added to the colors of the guides in $\Pi$, eliminates the problematic conflicts and makes $\Pi$ a $2 m_{1} m_{2}$-color guidance system as required.

\subsection{Binary queries.}

Lemma 5. Every binary regular query $\varphi[x, y]$ has witness functions of bounded guidance width.

Proof. Whenever a pair $(x, y)$ belongs to $\varphi(t)$, call the node $z=\operatorname{gca}(x, y)$ an $x$-intermediate node, and call $y$ a $z$-final node. We will define two guidance systems, the first one directing any source node $x$ to an $x$-intermediate one, and the second one directing any intermediate node $z$ to a $z$-final one. The two guidance systems will be combined using Lemma 4 .

A binary query is essentially a degenerate case of ternary query, with $y_{1}=y_{2}$. By Lemma 3 assume that $\varphi$ is path-based. Thus its truth value in a tree $t$ only depends on some regular properties of two words

$$
w_{1}=\operatorname{word}_{t}(x, \operatorname{gca}(x, y)) \text { and } w_{2}=\operatorname{word}_{t}(\operatorname{gca}(x, y), y),
$$

as depicted in the figure in Section 4.3 . (Words $w_{3}$ and $w_{4}$ are empty as $y_{1}=y_{2}$.) Namely, $(x, y)$ belongs to $\varphi(t)$ if and only if $\left(\alpha\left(w_{1}\right), \alpha\left(w_{2}\right)\right) \in F$, for a designated set $F \subseteq S^{2}$. Fix $\left(s_{1}, s_{2}\right) \in F$. We will define a guidance system for pairs $(x, y)$ which satisfy

$$
\alpha\left(w_{1}\right)=s_{1}, \quad \alpha\left(w_{2}\right)=s_{2} .
$$

(Then the required guidance system will be a disjoint union over all pairs $\left(s_{1}, s_{2}\right) \in F$.)

Assume further, without loss of generality, that $x$ is in the left subtree, and $y$ is in the right subtree of gca $(x, y)$, including possibly $y=\operatorname{gca}(x, y)$. (Again, the required guidance system will be a disjoint union of two systems.)

Consider deterministic word automata $\mathcal{A}_{1}$ and $\mathcal{A}_{2}$ that recognize the properties $\alpha^{-1}\left(s_{1}\right)$ and $\alpha^{-1}\left(s_{2}\right)$, respectively. Think of a run of $\mathcal{A}_{1}$, starting from a source node $x$, along the path in $t$ leading from $x$ to some $x$-intermediate node. Consider such runs of $\mathcal{A}_{1}$ starting from all source nodes $x$, one run from every source node. These runs may be translated into a guidance system, as follows.

Each of the runs labels nodes on the path from $x$ to an $x$-intermediate node with states of $\mathcal{A}_{1}$. The idea is that two source nodes $x$ and $x^{\prime}$ may be directed to the same intermediate node if the two runs of $\mathcal{A}_{1}$ that start in $x$ and $x^{\prime}$ label some node of $t$ with the same state. In other words, one may use the same guide both for $x$ and $x^{\prime}$. Thus there is a guidance system, with as many colors as the number of states of $\mathcal{A}_{1}$, that follows the runs of $\mathcal{A}_{1}$ until acceptance, and directs any source node $x$ to some $x$-intermediate node.

Likewise for $\mathcal{A}_{2}$, there is a corresponding guidance system, that leads any intermediate node $z$ to some $z$-final node. Applying Lemma 4 for these two guidance systems we get the result. 
4.6. Arrangement (A1). In this section we show that Theorem 2 holds if all triples selected by $\varphi\left[x, y_{1}, y_{2}\right]$ have arrangement $\mathrm{A} 1$, pictured below.

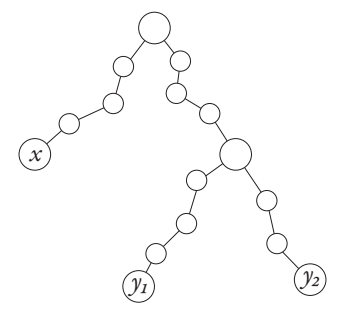

Suppose $\tau[x, y]$ is a binary query, and $\sigma\left[y, y_{1}, y_{2}\right]$ is a ternary query. We define the following ternary query

$$
\tau \circ_{y} \sigma\left[x, y_{1}, y_{2}\right] \quad=\quad \exists y \tau[x, y] \wedge \sigma\left[y, y_{1}, y_{2}\right] .
$$

Lemma 6. Let $\tau, \sigma$ be as above. If $\tau$ and $\sigma$ have bounded width witness functions then so does $\tau \circ_{y} \sigma$.

Proof. By considering the witness function for $\tau \circ_{y} \sigma$ obtained as a composition and applying Lemma 4 .

Lemma 7. We may assume without loss of generality that $\varphi$ only selects triples $\left(x, y_{1}, y_{2}\right)$ where $x=\operatorname{gca}\left(y_{1}, y_{2}\right)$.

Proof. By the considerations in Section 4.3, we know that a triple $\left(x, y_{1}, y_{2}\right)$ is selected by $\varphi$ if and only if the images, under the morphism $\alpha$, of the four path words $w_{1}, w_{2}, w_{3}, w_{4}$ belong to a designated set $F \subseteq S^{4}$ of accepting tuples.

Let $s_{1}, \ldots, s_{4} \in S$. Let $\tau_{s_{1}, s_{2}}$ be the binary query that selects a pair $(x, y)$ if

$$
\begin{aligned}
& \alpha\left(\operatorname{word}_{t}(\operatorname{gca}(x, y), x)\right)=s_{1} \\
& \alpha\left(\operatorname{word}_{t}(\operatorname{gca}(x, y), y)\right)=s_{2} .
\end{aligned}
$$

Likewise, let $\sigma_{s_{3}, s_{4}}$ be the ternary query that selects a triple $\left(y, y_{1}, y_{2}\right)$ if

$$
\begin{aligned}
\operatorname{gca}\left(y_{1}, y_{2}\right) & =y \\
\alpha\left(\operatorname{word}_{t}\left(y, y_{1}\right)\right) & =s_{3} \\
\alpha\left(\operatorname{word}_{t}\left(y, y_{2}\right)\right) & =s_{4} .
\end{aligned}
$$

The queries $\tau_{s_{1}, s_{2}}$ and $\sigma_{s_{3}, s_{4}}$ can be joined to define $\varphi$, in the following way.

$$
\varphi=\bigcup_{\left(s_{1}, s_{2}, s_{3}, s_{4}\right) \in F} \tau_{s_{1}, s_{2}} \circ_{y} \sigma_{s_{3}, s_{4}} .
$$

By Lemma 6, we see that the width of witness functions for $\varphi$ is bounded by the widths of the witness functions for the $\tau$ queries, which is bounded by Lemma 5 , and the width of the witness functions for the $\sigma$ queries. The latter are queries where the first variable is the gca of the second and third variables, which concludes the proof of the lemma. 
Thanks to the above lemma, we are left with a query $\varphi$ that selects triples in the arrangement pictured below (for future reference let us call this arrangement trivial).

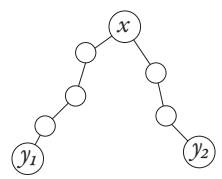

We will provide a 2-color guidance system that induces a witness function for $\varphi$ in $(t, E)$. This is guaranteed by the following lemma:

Lemma 8. Let $\varphi$ be any (not necessarily regular) query that selects only nodes in a trivial arrangement. Then $\varphi$ has witness functions of guidance width 2 .

Proof. The guidance system is constructed in a single root-to-leaf pass.

More formally, for each set $X$ of nodes that is closed under ancestors, we will provide a guidance system $\Pi_{X}$ that directs each node that is a source node and in $X$ to some witness, either $y_{1}$ or $y_{2}$. The guidance system will have the additional property that no tree edge is traversed by two guides.

The guidance system is constructed by induction on the size of $X$. The induction base, when $X$ has no nodes, is straightforward. We now show how $\Pi_{X}$ should be modified when adding a single $x$ node to $X$. When $x$ is not a source node, then nothing needs to be done. Otherwise, suppose that $x$ is a source node, and the witness is $\left(y_{1}, y_{2}\right)$. Since all guides in $\Pi_{X}$ originate in nodes from $X$, any guide that passes through $x$ must also pass through its parent. Using the additional assumption, we conclude that at most one guide $\pi$ from $\Pi_{X}$ passes through $x$. In particular, either the left subtree of $x$, which contains $y_{1}$, or the right subtree of $x$, which contains $y_{2}$, has no guide passing through it. We create a new guide that connects $x$ to the witness in the subtree without a guide.

For arrangement (A1) the proof of Theorem 2 is thus completed.

4.7. Arrangements $\mathrm{A} 2$ and $\mathrm{A} 3 \mathrm{~s}$. For the remaining arrangements, in this section we only show how they can be reduced to the simplified ones. We formulate Theorem 3 below, which we will use in this section, and which follows easily from the Main Lemma (forthcoming Lemma 9) to be proven in the next section.

To state the theorem, we need a new notion. A guidance system in a graph tree is called consistent wrt. a given ternary query if each of guides obeys the following uniqueness requirement: whenever a set $Z \subseteq X$ of source nodes is guided to the same node $y$, then there is a pair $\left(y_{1}, y_{2}\right)$ that is a witness pair for all nodes $Z$, with $y_{1}=y$ or $y_{2}=y$. Roughly speaking: if all nodes in $Z$ agree on the witness they are guided to, then they agree on the other witness as well. The notion of consistency is meaningful only relative to a given ternary query. Below, the consistency property will make it possible to combine two guidance systems appropriately.

Theorem 3. Every simplified regular query has witness functions of bounded guidance width. Furthermore, a consistent guidance system always exists (of the required bounded guidance width).

Now using Theorem 3 we prove Theorem 2 for arrangements $\mathrm{A} 2$ and $\mathrm{A} 3$. By symmetry, we only consider the arrangement A2]. We simplify the arrangement in two steps. 
First we claim that without loss of generality $x$ can be assumed to be an ancestor of $y_{2}$ - this may be shown by essentially the same technique as in Lemma 7 hence we omit the details. Second, we show that $y_{1}$ can be assumed to be an ancestor of $x$ and $y_{2}$. The arrangement (A2), as well as its two successive simplifications, are pictured below.
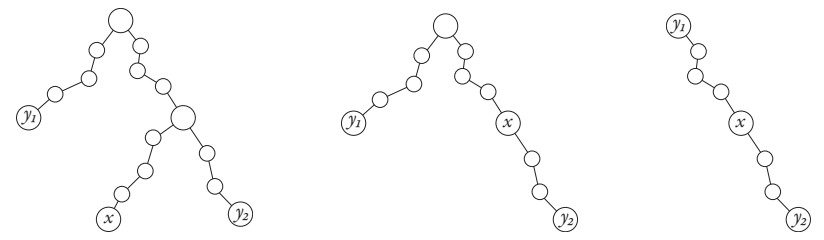

Let our starting arrangement be the middle one in the picture above, i.e. we assume that the first simplification has been already applied. Without loss of generality we may assume that $y_{1}$ is in the left subtree, and $x$ in the right subtree of the gca $\left(x, y_{1}\right)$ node (thus we again split into two sub-cases), and that both $x$ and $y_{1}$ are not equal to gca $\left(x, y_{1}\right)$.

By the considerations in Section 4.3, we know that a triple $\left(x, y_{1}, y_{2}\right)$ is selected by $\varphi$ in $t$ if and only if the images, under the morphism $\alpha$, of the three path words:

$$
\operatorname{word}_{t}\left(x, \operatorname{gca}\left(x, y_{1}\right)\right), \operatorname{word}_{t}\left(x, y_{2}\right), \operatorname{word}_{t}\left(\operatorname{gca}\left(x, y_{1}\right), y_{1}\right) \text {, }
$$

belong to a designated set $F \subseteq S^{3}$ of accepting tuples.

Fix $\left(s_{1}, s_{2}, s_{3}\right) \in F$. We define a guidance system for triples $\left(x, y_{1}, y_{2}\right)$ which satisfy

$$
s_{1}=\operatorname{word}_{t}\left(x, \operatorname{gca}\left(x, y_{1}\right)\right), s_{2}=\operatorname{word}_{t}\left(x, y_{2}\right), s_{3}=\operatorname{word}_{t}\left(\operatorname{gca}\left(x, y_{1}\right), y_{1}\right) \text {. }
$$

In general, the guidance system will be a disjoint union over all triples $\left(s_{1}, s_{2}, s_{3}\right) \in F$. Consider an arbitrary graph tree $(t, E)$ over which $\varphi$ is evaluated, together with an arbitrary subset $X$ of source nodes. We aim at constructing a guidance system whose number of colors depends only on $\varphi$, that directs any source node $x \in X$ to either $y_{1}$ or $y_{2}$, for some triple $\left(x, y_{1}, y_{2}\right)$ selected by $\varphi$. We will do it in two stages. In the first stage, the node $x$ is directed either to $y_{2}$, or to $y=\operatorname{gca}\left(x, y_{1}\right)$. By Theorem 3 we will be able to assume that this guidance system is consistent. In the second stage, every y node will be directed either to an appropriate $y_{1}$ node, or to the $y_{2}$ node, using Lemma 8 . Finally, we will compose the two guidance systems using Lemma 4.

Formally speaking, for the first stage we use the simplified query $\sigma_{s_{1}, s_{2}}\left[x, y, y_{2}\right]$ that selects a triple $\left(x, y, y_{2}\right)$ if

- $\alpha\left(\operatorname{word}_{t}(x, y)\right)=s_{1}$

- $\alpha\left(\operatorname{word}_{t}\left(x, y_{2}\right)\right)=s_{2}$

- $y<x \leq y_{2}$

- $x$ is in the right subtree of $y$.

The idea now is that the query $\sigma_{s_{1}, s_{2}}$ is evaluated over a modified graph tree $\left(t, E_{s_{3}}\right)$. The relation $E_{s_{3}}$ is defined as follows: $\left(y, y_{2}\right)$ is in $E_{s_{3}}$ iff $\left(y_{1}, y_{2}\right) \in E$ for some $y_{1}$ such that

- $y=\operatorname{gca}\left(y_{1}, y_{2}\right)$,

- $y_{1}$ is in the left subtree of $y$,

- $\alpha\left(\operatorname{word}_{t}\left(y, y_{1}\right)\right)=s_{3}$.

Intuitively, the first node $y_{1}$ of every edge $\left(y_{1}, y_{2}\right) \in E$ is moved to $y=\operatorname{gca}\left(y_{1}, y_{2}\right)$, but only if the equation $\alpha\left(\operatorname{word}_{t}\left(y, y_{1}\right)\right)=s_{3}$ holds. Note that we use here the more general notion of graph trees, rather than data trees, as the relation $E_{s_{3}}$ is not an equivalence in general. By Theorem 3 we know that $\sigma_{s_{1}, s_{2}}$ has a witness function induced by a consistent 
$m$-color guidance system $\Pi$, where $m$ only depends on $\sigma_{s_{2}, s_{3}}$ and does not depend on $t, E$ or $X$.

Each source node $x$ is directed so far either to some $y<x$, or to $y_{2} \geq x$. Without loss of generality we may assume that colors used for target nodes of the first kind are distinct from colors used for target nodes of the other kind. For the second stage, consider the set $Y$ of all target nodes $y$ of $\Pi$ of the first kind, and restrict attention to the induced sub-guidance system of $\Pi$. Note that every such node $y \in Y$ has an associated node $y_{1}$ located in the left subtree of $y$ such that $\alpha\left(\operatorname{word}_{t}\left(y, y_{1}\right)\right)=s_{3}$. Moreover, due to consistency of $\Pi, y$ has also an associated node $y_{2}$ located in the right subtree of $y$ such that $\alpha\left(\operatorname{word}\left(y, y_{2}\right)\right)=s_{1} s_{2}$. Consider the set of all such triples $\left(y, y_{1}, y_{2}\right)$ and apply Lemma 8 to obtain a 2-color guidance system that directs every $y \in Y$ to one of its two associated nodes.

Finally, using Lemma 4 we obtain a guidance system for $\varphi$ of bounded width.

As the graph tree $(t, E)$ and the subset $X$ were chosen arbitrarily, this completes the proof of Theorem 2 .

\section{Proof of Theorem 3}

Fix in this section a simplified regular query $\varphi\left[x, y_{1}, y_{2}\right]$, i.e., satisfying the conditions (1) and (2) from Section 4. Since the query is regular, the dependency stated in item (2) is a regular dependency. We may thus assume a semigroup morphism $\alpha: \Sigma^{*} \rightarrow S$ recognizing $\varphi$, which maps each word to an element of a finite semigroup $S$. Whether or not a triple $\left(x, y_{1}, y_{2}\right)$ is selected by $\varphi$ depends only on the images

$$
\begin{aligned}
& s_{1}=\alpha\left(\operatorname{word}_{t}\left(y_{1}, x\right)\right) \in S \\
& s_{2}=\alpha\left(\operatorname{word}_{t}\left(x, y_{2}\right)\right) \in S .
\end{aligned}
$$

In other words, there is a set of accepting pairs $F \subseteq S^{2}$ such that $\varphi(w)$ is the set of triples $\left(x, y_{1}, y_{2}\right)$ with $\left(s_{1}, s_{2}\right) \in F$. We fix the morphism $\alpha$ for the rest of this section.

We distinguish two types of edges in a graph tree $(t, E)$. The tree edges are edges that connect parents with children, as well as a dummy edge going into the root of the tree and dummy edges going out of the leaves. The class edges are the edges from $E$. We order tree edges by the ancestor relation $\leq$, according to the positions in the tree, with the dummy edges coming as the least one and the maximal ones, respectively. For two tree edges $e \leq f$ in a tree $t$, we write $\operatorname{word}_{t}(e, f)$ for the word labeling $t$ on the path that begins in the target of $e$ and ends in the source of $f$. In particular $\operatorname{word}_{t}(e, e)=\epsilon$.

Forward Ramseyan splits. The key tool in our proof is a forward Ramseyan split, as defined by Colcombet in [6]. Let $t$ be a tree labeled with $\Sigma$. A split of height $n$ in $t$ is a function $\sigma$ that maps each tree edge to a number in $\{1, \ldots, n\}$. We say that two tree edges $e<f$ are neighbors with respect to a split $\sigma$, if $\sigma$ assigns the same number to $e$ and $f$, and all tree edges between $e$ and $f$ are mapped to at most $\sigma(e)$. A split $\sigma$ is called forward Ramseyan with respect to a morphism $\alpha$ if

$$
\alpha\left(\operatorname{word}_{t}(e, f)\right)=\alpha\left(\operatorname{word}_{t}(e, g)\right)
$$

holds for every three pairwise neighboring tree edges $e<f<g$. The following theorem was shown in [6]. 
Theorem 4. Fix a morphism $\alpha: \Sigma^{*} \rightarrow S$. Every tree $t$ has a forward Ramseyan split of height $\mathcal{O}(|S|)$. Furthermore, the split is top-down deterministic in the sense that all tree edges from a node to its children are assigned the same number in the split.

From now on wlog we consider only complete binary trees, where each non-leaf node has precisely two children.

Factors. Two comparable wrt. $\leq$ (i.e., belonging to one path) tree edges $e$ and $f$ are called visible if all tree edges between $e$ and $f$ are mapped by the split to values strictly smaller than $\sigma(e)$ and $\sigma(f)$. Visible pairs of tree edges naturally determine a nested factorization of $t$ in the following way.

A pre-factor in a tree $t$ is a connected set of nodes (connected by tree edges) such that if a node $x$ is in the pre-factor, then either all children of $x$ are in the pre-factor, or none of them. Each pre-factor of $t$ has a root and some leaves (maximal nodes wrt. $\leq$ ), and inherits its edges from $t$. In the definitions below, we talk about tree edges and not class edges. We distinguish internal edges of a pre-factor, connecting two nodes in that pre-factor, and external edges connecting the root or the leaves with some node outside the pre-factor. This includes the tree edge leading to the root of the pre-factor (called the root edge of the pre-factor) and the tree edges going out of the leaves (called the leaf edges of the pre-factor). Note that external edges may be either proper tree edges, or dummy edges. As the split $\sigma$ is assumed to be deterministic, all tree edges leaving a given leaf of a pre-factor are assigned the same number. A pre-factor $F$ is called a factor in $t$ if it respects the split $\sigma$ in the following way: the root edge is visible from each of the leaf edges. This means that on each (shortest) path in a factor from its root edge to a leaf edge, numbers assigned by $\sigma$ to the internal edges on that path are strictly smaller than those assigned to the two external edges. By the height of a factor we mean the greatest number assigned to an internal edge, or 0 if no such edge exists (the case of one-node pre-factor). Additionally, the whole tree $t$ is also a factor if, wlog, we assume that the root dummy edge is visible from all leaf dummy edges; its height at most equals the height of $\sigma$.

A subfactor of a factor $F$ is any factor $G \subsetneq F$ that is maximal with respect to inclusion. By the definition of factor, we get:

Claim 1. Every two different subfactors of $F$ are disjoint (have disjoint sets of nodes, but possibly share an external edge).

Proof. Indeed, assume two non-disjoint different subfactors $F_{1}, F_{2}$ of some factor $F$. The root node of one of them, say the root node $r_{1}$ of $F_{1}$, is necessarily contained in the other. As $F_{1}$ is not included in $F_{2}$, there must be a leaf node $l_{1}$ of $F_{1}$ not contained in $F_{2}$; the path that leads to that leaf node passes though a leaf node $l_{2}$ of $F_{2}$. If we denote by $r_{2}$ the root node of $F_{2}$ we know that the four nodes are located on one path in the following order:

$$
r_{2} \leq r_{1} \leq l_{2}<l_{1}
$$

This arrangement of the nodes is in a clear contradiction with the assumption that the tree edge incoming to $r_{i}$ is visible from (all) tree edges outgoing from $l_{i}$, for $i=1,2$.

Hence each factor $F$ is the disjoint union of its subfactors. We say a subfactor $G$ is an ancestor of a subfactor $H$ if their roots are so related. Likewise we talk about a subfactor being a child or parent of some other subfactor.

A factor of height 2 together with its decomposition into subfactors is pictured below. 

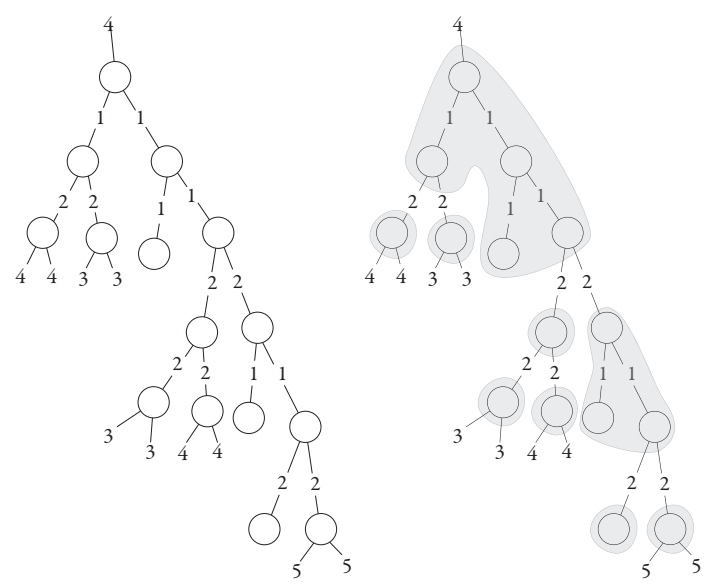

Our proof of Theorem 3 is based on the Main Lemma stated below. The lemma is proved by induction on the height of factors. To state the lemma recall the notion of consistent guidance system introduced in Section 4.7 .

Lemma 9 (Main Lemma). Fix a factor height $h$. There is a bound $n \in \mathbb{N}$, depending only on $\varphi$ and $h$, such that for every graph tree $(t, E)$, every factor $F$ in $t$ of height $h$, and every set $X \subseteq F$ of source nodes, there is a witness function for $\varphi$ and $X$ in $(t, E)$ induced by a consistent guidance system using at most $n$ colors. Furthermore, this witness function only points to descendants of the root of $F$.

The proof of the lemma is by induction on the height $h$. The number of colors $n$ will depend on $h$ and the size of the monoid $S$ recognizing the query. It will not depend on $t$. When going from height $h$ to height $h+1$, there will be a quadratic blowup in the number of colors. Therefore, $n$ will be doubly exponential in the height of $F$.

Since the witness function will be induced by a guidance system, the last assumption in Lemma 9 could be restated as saying that no guide passes through the root edge of $F$. Theorem 3 is a special case of the Main Lemma when $F$ is the whole tree.

The base case when $h=0$, and hence the factor $F$ has one or no nodes, is easy (1 color, going downwards, is sufficient). For the induction step, fix a factor $F$, and assume that there is a bound $n$ sufficient for any factor of smaller height than $F$, which includes all subfactors of $F$. Below, subfactors of $F$ are simply called subfactors, without explicitly referring to $F$.

A tree edge of $F$ that is an external edge of one if its subfactors is called a border edge. In particular each external edge of $F$ is a border edge. Special care will be paid in our proof to internal (i.e. not external) border edges, i.e., the edges that connect one subfactor to another.

Claim 2. If two internal border edges in a factor are comparable by the ancestor relation $\leq$ then they are assigned the same value by the split.

Proof. Assume two internal border edges $e<f$, with different values assigned by the split, such that no other internal border edge is located on the (shortest) path from $e$ to $f$. As values assigned to all external border edges are strictly larger, one of $e, f$ is visible from some (possibly external) border edge "over" the other. That is, either $e$ is visible from some $e^{\prime}>f$, or $f$ is visible from some $f^{\prime}<e$. In both cases, one of $e, f$ is an internal edge of some subfactor - a contradiction. 
We do a case distinction, regarding the number of internal border edges on the paths from a source to witness nodes. For a node $x \in X$ and a witness $\left(y_{1}, y_{2}\right)$ we define two numbers $m_{1}, m_{2}$. Let $m_{1}$ be the number of internal border edges on the path between $y_{1}$ and $x$, and let $m_{2}$ be the number of internal border edges on the path between $x$ and $y_{2}$. For technical convenience, we deliberately choose not to count external border edges. We divide the set $X$ into three parts:

$X_{1} \quad$ Nodes $x \in X$ that have a witness with $m_{2} \leq 1$.

$X_{2} \quad$ Nodes $x \in X$ that have a witness with $m_{1} \leq 1$ and $m_{2} \geq 2$.

$X_{3}$ Nodes $x \in X$ that have a witness with $m_{1}, m_{2} \geq 2$.

We prove the Main Lemma for each of the three parts separately. Next, we combine the three guidance systems into a single guidance system. Our construction will yield two kinds of guides: the ancestor guides pointing to the first witness and thus going up a tree; and descendant guides pointing to the second witness, and thus going down the tree. Interestingly, ancestor guides will be only created in case of nodes from $X_{2}$. All the guides will satisfy the consistency condition required in Lemma 9.

Nodes from $X_{1}$, i.e. nodes that have a witness with $m_{2} \leq 1$. Consider a subfactor $G$ of $F$. In this case, each node $x \in X_{1} \cap G$ has a descendant witness $y_{2}$ that is either in $G$, or in a child subfactor of $G$, or perhaps outside $F$. Apply the induction assumption to $G$, producing a guidance system $\Pi_{G}$ with at most $n$ colors. Since the Main Lemma requires the guidance system to point to descendants of the factor's root, and $m_{2} \leq 1$, we infer that inside $F$ the guides of $\Pi_{G}$ can only intersect $G$ and its child subfactors, and no other subfactors (it is possible that the guides leave the factor $F$, though). Therefore, all the guidance systems $\Pi_{G}$, for all subfactors $G$ of $F$, can be combined into a single guidance system with at most $2 n$ colors, used alternatingly for even and odd depths.

Nodes from $X_{2}$, i.e. nodes that have a witness with $m_{1} \leq 1$ and $m_{2} \geq 2$. In this case, for each node $x \in X_{2}$ there is an ancestor witness $y_{1}$ that is either in the subfactor of $x$, in the parent subfactor, or outside $F$. Note that the latter is possible only when $x$ belongs either to the root subfactor of $F$, or to some of its child subfactors; denote this set of subfactors by $\mathcal{G}_{0}$. We will construct the guidance system in a step-by-step manner, for all subfactors, according to the ancestor ordering.

Formally speaking, consider a family $\mathcal{G}$ of subfactors that is closed under ancestors and includes $\mathcal{G}_{0}$. We provide a guidance system $\Pi_{\mathcal{G}}$ of $4 n^{2}+3 n$ colors that provides witnesses for all nodes of $X$ belonging to the subfactors in $\mathcal{G}$. The construction of $\Pi_{\mathcal{G}}$ is by induction on the number of subfactors in $\mathcal{G}$.

The induction base is when $\mathcal{G}$ equals $\mathcal{G}_{0}$. For each subfactor $G \in \mathcal{G}_{0}$, we apply the Main Lemma, for the smaller height, to $G$ and nodes from $X_{2}$ that belong to $G$, yielding an $n$-color guidance system. We combine these guidance systems into $\Pi_{\mathcal{G}_{0}}$ as follows: use one set of $n$ colors for the root subfactor, and another set of $n$ colors for all the child subfactors of the root.

For the induction step, suppose that we have already constructed $\Pi_{\mathcal{G}}$ for $\mathcal{G}$, and that $G \notin \mathcal{G}$ is a subfactor whose parent is in $\mathcal{G}$. Consider the guides of $\Pi_{\mathcal{G}}$ that pass through the root edge of $G$. We apply two distinctions to these guides. First, we use the name parent guides, for the guides that originate in the parent subfactor of $G$, and the name far guides for the other guides. Second, we use the name ending guides for the guides whose target is in $G$ and the name transit guides for the other guides, which continue into a child subfactor 
of $G$, or even exit $F$. Altogether, there are four possibilities: parent transit guides, far ending guides, etc. We assume additionally that there are at most $n$ parent guides and at most $2 n$ far and parent guides altogether, and hence at most $2 n$ guides entering $G$. This additional invariant is satisfied by the induction base, and it will be preserved through the construction.

Apply the induction assumption of the Main Lemma to $G$ and nodes from $X_{2}$ that belong to $G$, yielding a guidance system $\Pi$ with $n$ fresh colors. We use the name starting guides for the guides of $\Pi$. We want to combine $\Pi_{\mathcal{G}}$ with $\Pi$ in such a way that the resulting guidance system still uses at most $4 n^{2}+3 n$ colors, like $\Pi_{\mathcal{G}}$, and satisfies the additional invariant. If we were to simply take the two systems together, we might end up with a leaf edge of $G$ which is traversed both by starting and transit guides, which could exceed the bound $2 n$ on guides passing through border edges.

We solve this problem as follows. Consider the leaf edges of $G$ that are traversed by the far transit guides. There are at most $2 n$ such edges by our invariant assumption. We will remove all starting guides that pass any of these edges, and find other witnesses for nodes that use these starting guides. This guarantees that the invariant condition is recovered: at most $2 n$ guides passes through any leaf edge of $G$, and at most $n$ of them are starting guides. These other witnesses will be ancestors. This explains why the induction starts with $\mathcal{G}$ containing the root subfactor and its children, since these are the subfactors that may have ancestor witnesses outside the whole factor $F$ (recall that passing through the root edge is not counted in $m_{1}$ ). The statement of the Main Lemma does not allow guides that pass through the root edge of $F$.

The removing of starting guides proceeds as follows. Let $e$ be a leaf edge of $G$ that is traversed by a far transit guide, which has color $j$ in the guidance system $\Pi_{\mathcal{G}}$. Let $\pi$ be a starting guide, which has color $i$ in $\Pi$, that also traverses $e$, with $y_{2}$ its target node. By the consistency property of $\pi$, there is some $y_{1}$ such that $\left(y_{1}, y_{2}\right) \in E$ is a witness pair for all source nodes of $\pi$. Note that by assumption on $m_{1} \leq 1$, the node $y_{1}$ is either in the subfactor $G$ or its parent. We create an ancestor guide with a fresh color that connects all the source nodes of $\pi$ to $y_{1}$. The color of this guide, which we call an ancestor color, will take into account three parameters: the colors $i$ and $j$, as well as a parity bit $b \in\{0,1\}$. The parity bit is 0 if and only if $G$ has an even number of ancestor subfactors. We use the triple $(i, j, b)$ for the color name.

We will show that this new ancestor guide does not conflict with any other ancestor guide with the same color. Each new ancestor guide is contained in $G$ and possibly its parent subfactor $H$, by assumption on $m_{1} \leq 1$. Inside the subfactor $G$ there is at most one ancestor guide of each color, so there are no collisions inside $G$. One could imagine, though, that a new ancestor guide $\pi$ with color $(i, j, b)$ collides inside the parent subfactor $H$ with some other ancestor guide $\pi^{\prime}$ of the same color. Since the colors of $\pi$ and $\pi^{\prime}$ agree on the parity bit $b$, we conclude that the guide $\pi^{\prime}$ cannot originate in $H$, which has a different parity than $G$. Therefore, $\pi^{\prime}$ must originate in some other child subfactor of $H$, call it $G^{\prime}$, that had been previously added to $\mathcal{G}$. Since the color of $\pi^{\prime}$ is also $(i, j, b)$, we conclude that $j$ was the color of a far transit guide in $G^{\prime}$. This is impossible, since a far transit guide in $G^{\prime}$ or $G$ must originate not in $H$, but in an ancestor of $H$, and therefore there would be a collision in the root of $H$.

The ancestor guides created above are the only ancestor guides in our solution. In the subfactors where they are used, the ancestor guides have the target in the parent subfactor. 
Let us count the number of colors used. We need $2 n$ colors for the transit guides, and $n$ colors for the starting guides. For the ancestor guides, we need $4 n^{2}$ colors. Altogether, we need $4 n^{2}+3 n$ colors. Note that all the guides satisfy the consistency condition required by Lemma 9 .

Nodes from $X_{3}$, i.e. nodes that have a witness with $m_{1}, m_{2} \geq 2$. In this case, each node $x \in X_{3}$ has a witness $\left(y_{1}, y_{2}\right)$ such that the path from $y_{1}$ to $x$, as well as the path from $x$ to $y_{2}$, passes through at least two internal border edges. This case is the only one where we use the forward Ramseyan split.

Consider a source $x$ with a witness $\left(y_{1}, y_{2}\right)$. The internal border edges naturally split $\operatorname{word}_{t}\left(y_{1}, x\right)$ and $\operatorname{word}_{t}\left(x, y_{2}\right)$ into $m_{1}+1$ and $m_{2}+1$ words, respectively:

$$
\begin{aligned}
\operatorname{word}_{t}\left(y_{1}, x\right) & =v_{0} \cdot v_{1} \cdot \ldots \cdot v_{m_{1}} \\
\operatorname{word}_{t}\left(x, y_{2}\right) & =w_{0} \cdot w_{1} \cdot \ldots \cdot w_{m_{2}} .
\end{aligned}
$$

The first letter of $v_{0}$ is the label of $y_{1}$. The last letter of $v_{m_{1}}$ and also the first letter of $w_{0}$ is the label of $x$. The last letter of $w_{m_{2}}$ is the label of $y_{2}$ (recall that $y_{1}$ or $y_{2}$ might be outside $F$ ). Furthermore, each two consecutive internal border edges are not only visible, but also neighbouring, by Claim 2. Hence, as we have a forward Ramseyan split (cf. (5.3)), the values $\alpha\left(\operatorname{word}_{t}\left(y_{1}, x\right)\right)$ and $\alpha\left(\operatorname{word}_{t}\left(x, y_{2}\right)\right)$ are determined by the first two parts and the last part:

$$
\begin{aligned}
& \text { (i) } \alpha\left(\operatorname{word}_{t}\left(y_{1}, x\right)\right)=\alpha\left(v_{0}\right) \cdot \alpha\left(v_{1}\right) \cdot \alpha\left(v_{m_{1}}\right) \\
& \text { (ii) } \alpha\left(\operatorname{word}_{t}\left(x, y_{2}\right)\right)=\alpha\left(w_{0}\right) \cdot \alpha\left(w_{1}\right) \cdot \alpha\left(w_{m_{2}}\right) .
\end{aligned}
$$

Let us fix six values $s_{1}, \ldots, s_{6} \in S$. By splitting the set $X_{3}$ into at most $|S|^{6}$ parts, each requiring a separate guidance system, we can assume that each $x \in X_{3}$ has a witness where

$$
\begin{array}{lll}
s_{1}=\alpha\left(v_{0}\right) & s_{2}=\alpha\left(v_{1}\right) & s_{3}=\alpha\left(v_{m_{1}}\right) \\
s_{4}=\alpha\left(w_{0}\right) & s_{5}=\alpha\left(w_{1}\right) & s_{6}=\alpha\left(w_{m_{2}}\right) .
\end{array}
$$

We will only consider witnesses that satisfy the assumptions above.

We now proceed to create the guidance system. As in the case $m_{1} \leq 1$, the guidance system will be defined for a family $\mathcal{G}$ of subfactors that is closed under ancestors. The guidance system will use at most 3 colors, and will have the following additional invariant property: if $e$ is an edge that connects a subfactor $G$ with a child subfactor $H$, then at most two guides pass through $e$. Furthermore, if exactly two guides pass through $e$, then one of the guides has its target in $H$.

The construction is by induction on the number of subfactors in $\mathcal{G}$. The induction base when $\mathcal{G}$ has no subfactors is obvious. Below we show how to modify a guidance system $\Pi_{\mathcal{G}}$ for $\mathcal{G}$ when adding a new subfactor $G$.

Consider the (at most two) guides of $\Pi_{\mathcal{G}}$ that pass through the edge connecting $G$ to $\Pi_{\mathcal{G}}$. As in the case $m_{1} \leq 1$, we use the term transit guide for the guides of $\Pi_{\mathcal{G}}$ that enter $G$ through its root and exit through one of its external leaf edges. By the invariant assumption, there is at most one transit guide.

We now define a guidance system $\Pi$ for the nodes in $G$, which we will next combine with $\Pi_{\mathcal{G}}$.

Claim 3. There is a one-color guidance system $\Pi$ defining a witness function for all nodes in $G \cap X_{3}$. 
Proof. For each node $x \in G \cap X_{3}$, choose the lexicographically first witness $y_{2}>x$ that satisfies the assumptions on the six images in the semigroup, call it $y_{x}$. Let $Y$ be all these witnesses $y_{x}$, for $x \in G \cap X_{3}$; this set is an antichain with respect to the descendant relation. For each $y \in Y$, let $X_{y}$ be the chain of nodes $x$ which are witnessed by $y$. By the lexicographic assumption, if $y, y^{\prime} \in Y$ are such that $y$ is lexicographically before $y^{\prime}$, then no element from $X_{y}$ has an ancestor in $X_{y^{\prime}}$. Consequently, if we define $\pi_{y}$ to be the guide that connects all $X_{y}$ to $y$, then $\Pi=\left\{\pi_{y}\right\}_{y \in Y}$ is a one-color guidance system for all nodes in $G \cap X_{3}$.

The guides of $\Pi$ we call starting guides as usual.

We now need to combine $\Pi$ and $\Pi_{\mathcal{G}}$. If we simply combine $\Pi_{\mathcal{G}}$ and $\Pi$, we might end up with a starting guide going through and external edge of $G$ that is already traversed by two transit guides. To avoid this problem, we need to do an optimisation relying on a simple observation formulated in the claim below.

A descendant guide $\pi$ is called live in a subfactor $G$ if $\pi$ passes through $G$, and its target is not in a child subfactor of $G$ (i.e., the target is in a proper descendant of some child of $G$, or outside $F$ ). The idea is that the target of $\pi$ satisfies the assumption $m_{2} \geq 2$ from the 'point of view' of nodes in $G$. Note that guides live in $G$ may be either transit or starting in $G$.

Claim 4. Suppose that two consistent descendant guides $\pi$ and $\pi^{\prime}$ are live in a subfactor $G$ and exit $G$ through the same edge. Suppose also that at least one of them is starting in $G$. Then all source nodes of one of $\pi, \pi^{\prime}$ can be moved to the other.

Proof. Let $\left(y_{1}, y_{2}\right)$ be the witness pair corresponding to $\pi$ and let $\left(y_{1}^{\prime}, y_{2}^{\prime}\right)$ be the witness pair corresponding to $\pi^{\prime}$ - by consistency, not only the second witnesses $y_{2}$ and $y_{2}^{\prime}$ are determined by $\pi$ and $\pi^{\prime}$, but the whole witness pairs. Note that the source nodes of a descendant guide in $G$ are all situated on one path from the root of $G$ to one of the leaves. If both $\pi$ and $\pi^{\prime}$ are starting in $G$, assume wlog that $\pi$ has a source node that is an ancestor of all source nodes of $\pi^{\prime}$; otherwise one of the guides is starting in $G$, wlog assume it is $\pi^{\prime}$. As only the case of $m_{1}, m_{2} \geq 2$ is considered, and the values $s_{1}, \ldots, s_{6}$ are fixed, due to equation (5.4)(i) the pair $\left(y_{1}, y_{2}\right)$ is a witness for all source nodes of $\pi^{\prime}$ as well. Thus, these nodes may be guided to $y_{2}$ instead of $y_{2}^{\prime}$.

We use the term live transit guide for the transit guides that are live in $G$, and dead transit guide for the other transit guides (those that have their target in a child subfactor of $G)$.

Consider an edge $e$ that connects $G$ with a child subfactor $H$. Suppose first that $e$ is traversed by a starting guide and a live transit guide. Using the claim we merge the starting guide with the transit one. Therefore, we end up satisfying the invariant property: $e$ is passed by at most one live guide, and possibly by one dead transit guide.

We have thus completed the proof of the Main Lemma and thus also of Theorem 3 .

\section{Applications}

In this section, we present two applications of our results. The first application is a class of XML documents for which emptiness of XPath is decidable. The second application is a proof that two-variable first-order logic is not captured by XPath, in the presence of two attribute values per node. 
Satisfiability of XPath. As we said in the introduction, our study on class automata is a first step in a search for structural restrictions on data words and data trees which make XPath satisfiability decidable.

One idea for a structural restriction would be a variant of bounded clique width, or tree width. Maybe bounded clique or tree width are interesting restrictions, but they are not relevant in the study of class automata. This is because bounded clique width or tree width, when defined in the natural way for data trees, guarantees decidable satisfiability for a logic far more powerful than class automata: MSO with navigation and equal data value predicates.

Here we provide a basic example of a restriction on inputs that works for class automata but not for MSO. A data tree is called bipartite (bipartite refers to the data) if its nodes can be split into two connected (by the child relation) sets $X, Y$ such that every class has at most one node in $X$ and at most one node in $Y$.

Satisfiability of MSO, or even FO, with navigation and data equality predicates is undecidable even for bipartite data words. For instance, a solution to the Post Correspondence Problem can be encoded in a bipartite data word using a FO formula.

This coding, however, cannot be captured by class automata, which is implied by the following theorem.

Theorem 5. On bipartite data trees, emptiness is decidable for class automata, and therefore also for XPath.

Proof. The key insight is that data trees which use each data value at most twice can be described using semilinear sets. To avoid notational complications, assume that every data value appears exactly twice in a bipartite tree.

Consider a class automaton $\mathcal{A}$ over input alphabet $\Sigma$. Suppose that the work alphabet is $\Gamma$, and the transducer is $f$. Let the class condition be a language over alphabet $\Gamma \times\{0,1\}$, recognized by a deterministic bottom-up tree automaton $\mathcal{C}$, with states $Q$. For technical convenience, assume that a run of $\mathcal{C}$ labels tree edges, instead of tree nodes, with states. Thus all leaf dummy edges of an input tree $u$ over $\Gamma \times\{0,1\}$ are labeled with the initial state of $\mathcal{C}$, and the labeling of all other edges is uniquely determined by $u$. Let $\mathcal{C}(u)$ denote the state that labels the root dummy edge of $u$. A tree $u$ is accepted if $\mathcal{C}(u)$ is an accepting state.

By assumption, the nodes of a bipartite data tree $(t, \sim)$ are partitioned into two connected subsets, and thus there is a single edge that splits the two subsets; call this edge the border edge. Such a tree $t$ with a distinguish edge may be modeled as $t \otimes\left\{z, z^{\prime}\right\}$, where $z$ and $z^{\prime}$ are the two nodes connected by the border edge. Further, $t$ may be split into two smaller trees, according to the partition: the border edge is a dummy root edge for one of the trees, and a leaf dummy edge for the other one. Call these two induced trees lower and upper tree, respectively.

For the lower tree of $t$, call it $t_{l}$, and a subset $X$ of nodes of $t_{l}$, it makes sense to write $\mathcal{C}\left(t_{l} \otimes X\right)$. For the upper one, call it $t_{u}$, we will need a slightly different notation. Assume that the automaton $\mathcal{C}$ reads $t_{u} \otimes X$, for some $X$, starting in the initial state in all dummy leaf edges of $t_{u}$ except for the border edge, where the automaton starts in some chosen state $q$. If $\mathcal{C}$ accepts the tree $t_{u} \otimes X$ under this assumption, we write $q \in \mathcal{C}^{-1}\left(t_{u} \otimes X\right)$. In particular, observe that the automaton $\mathcal{C}$ accepts $t \otimes X$ if and only if $\mathcal{C}\left(t_{l} \otimes X_{l}\right) \in \mathcal{C}^{-1}\left(t_{u} \otimes X_{u}\right)$, where $X=X_{l} \cup X_{u}$ is the induced partition of $X$. 
Given a tree $t$ over $\Gamma$ with a single distinguished edge, say $t \otimes\left\{z, z^{\prime}\right\}$, let $\pi\left(t \otimes\left\{z, z^{\prime}\right\}\right)$ be the set of all trees $s$ over $Q \times\{l, u\}$ that satisfy the following conditions:

- the set of nodes of $s$ is the same as the set of nodes of $t$,

- if a node $x$ of $s$ is in $t_{l}$ then it is labeled by $\left(\mathcal{C}\left(t_{l} \otimes\{x\}\right), l\right)$,

- if a node $x$ of $s$ is in $t_{u}$ then it is labeled by $(q, u)$, for some $q \in \mathcal{C}^{-1}\left(t_{u} \otimes\{x\}\right)$.

Using $\pi$, we define the relation $\sigma$ between trees over $\Gamma$ and trees over $Q \times\{l, u\}$ as follows: $(t, s) \in \sigma$ if $s \in \pi\left(t \otimes\left\{z, z^{\prime}\right\}\right)$ for some edge $\left(z, z^{\prime}\right)$ in $t$.

Claim 5. The relation $\sigma$ is computable by a nondeterministic letter-to-letter transducer.

Let $T_{\Sigma}$ denote the set of all trees over $\Sigma$. As nondeterministic transducers preserve regular languages, we have:

Claim 6. Both $K=f\left(T_{\Sigma}\right)$ and $\sigma(K)=\{s:(t, s) \in \sigma, t \in K\}$ are effectively computable regular languages over $\Gamma$ and $Q \times\{l, u\}$, respectively.

Claim 7. A class automaton $\mathcal{A}$ accepts some bipartite data tree if and only if $\sigma(K)$ contains a tree that satisfies, for every $q \in Q$, the following condition: the number of nodes labeled by $(q, l)$ is the same as the number of nodes labeled by $(q, u)$.

With the last two observations decidability follows immediately. To decide emptiness of a given class automaton $\mathcal{A}$, compute the Parikh image of the language $\sigma(K)$, an effectively semi-linear set by Claim 6, intersect this set with the semi-linear condition of Claim 7 , and ckeck for emptiness of the resulting semi-linear set.

Multiple attributes. Heretofore, we have studied data trees, which model XML documents where each node has one data value. In this section, and this one only, we consider the situation where each node $x$ has $n$ data values. Formally, an $n$-data tree consists of a tree $t$ over the finite alphabet and functions $d_{1}, \ldots, d_{n}$ which map the tree nodes to data values. How does XPath deal with multiple data values? Instead of $y_{1} \sim y_{2}$ and $y_{1} \nsim y_{2}$, we can use any formula of the form

$$
d_{i}\left(y_{1}\right)=d_{j}\left(y_{2}\right) \quad \text { where } i, j \in\{1, \ldots, n\}
$$

or its negation (for inequality). For $n>1$ we need more information than just the partitions of nodes into classes of $\sim_{i}$, for each $i \in\{1, \ldots, n\}$. An example is the property "every node has the same data value on attributes 1 and 2 ".

How do we extend class automata to read $n$-data trees? For one data value, the class condition is a language over the alphabet $\Gamma \times\{0,1\}$. For $n$ data values, the class condition is a language over the alphabet $\Gamma \times\{0,1\}^{n}$. An $n$-data tree $\left(t, d_{1}, \ldots, d_{n}\right)$ is accepted if there is an output $s$ of the transducer on $t$ such that for every data value $d$, the tree

$$
s \otimes d_{1}^{-1}(d) \otimes \cdots \otimes d_{n}^{-1}(d)
$$

is accepted by the class condition. By the same technique as in the proof of Theorem 1, we can prove that the automata capture XPath.

A consequence is that for $n \geq 2$, XPath does not capture two-variable first-order logic (unlike the case of $n=1$ ). This was an open question.

Theorem 6. The following (two-variable) property

$$
\psi=\forall x \forall y \quad d_{1}(x)=d_{1}(y) \Longleftrightarrow d_{2}(x)=d_{2}(y)
$$

cannot be defined by a boolean query of XPath. 
Proof. Towards a contradiction, suppose that $\psi$ is recognized by any class automaton in the generalized version for 2 data values. The document that will confuse the automaton will be a word. Consider the document (over a one letter alphabet) with positions $1, \ldots, 2 n$, where the data values are defined

$$
\begin{aligned}
d_{1}(i)=d_{1}(n+i)=i & \text { for } i \in\{1, \ldots, n\} \\
d_{2}(i)=d_{2}(n+i)=-i & \text { for } i \in\{1, \ldots, n\}
\end{aligned}
$$

Since the above document satisfies $\psi$, it should also be accepted by the automaton. Let the work alphabet of the automaton be $\Gamma$, and let $a_{1} \cdots a_{2 n} \in \Gamma^{*}$ be the word produced by the automaton in the accepting run. For a data value $d$, we use the term class string of $d$ for the word

$$
a_{1} \cdots a_{2 n} \otimes d_{1}^{-1}(d) \otimes d_{2}^{-1}(d) .
$$

By definition of the way class automata accept documents, each class string should belong to the class condition. Consider a number $i \in\{1, \ldots, n\}$. The class string of $i$ is

$$
u_{i}=a_{1} \cdots a_{2 n} \otimes\{i, n+i\} \otimes \emptyset
$$

Suppose that

$$
\alpha:(\Gamma \times\{0,1\} \times\{0,1\})^{*} \rightarrow S
$$

is a morphism recognizing the class condition. If $n$ is greater than $|S|^{2}$, then we can find two data values $i<j \in\{1, \ldots, n\}$ such that

$$
\begin{aligned}
\alpha\left(u_{i} \mid\{1, \ldots, n\}\right) & =\alpha\left(u_{j} \mid\{1, \ldots, n\}\right) \\
\alpha\left(u_{i} \mid\{n+1, \ldots, 2 n\}\right) & =\alpha\left(u_{j} \mid\{n+1, \ldots, 2 n\}\right) .
\end{aligned}
$$

Consider a new document obtained from the previous one by swapping the first, but not second, data value in the positions $i$ and $j$. This new document violates the property $\psi$. To get the contradiction, we will construct an accepting run of the class automaton for this new document. The output of the transducer is the same $a_{1} \cdots a_{2 n}$. The class strings are the same for data values other than $i$ and $j$, so they are also accepted. For the class strings of $i$ and $j$, the images under $\alpha$ are the same by assumption on $i$ and $j$, and hence they are also accepted.

\section{ACKNOWLEDGMENT}

The authors would like to thank the anonymous reviewers for their valuable comments.

\section{REFERENCES}

[1] Michael Benedikt, Wenfei Fan, and Floris Geerts. XPath satisfiability in the presence of DTDs. J. $A C M$, $55(2), 2008$.

[2] H. Björklund and T. Schwentick. On notions of regularity for data languages. In FCT, pages 88-99, 2007.

[3] M. Bojańczyk and S. Lasota. An extension of data automata that captures XPath. In Proc. LICS'10, pages 243-252, 2010.

[4] M. Bojańczyk, A. Muscholl, T. Schwentick, and L. Segoufin. Two-variable logic on data trees and XML reasoning. J. ACM, 56(3), 2009.

[5] Mikolaj Bojanczyk, Claire David, Anca Muscholl, Thomas Schwentick, and Luc Segoufin. Two-variable logic on data words. ACM Trans. Comput. Log., 12(4):27, 2011. 
[6] T. Colcombet. A combinatorial theorem for trees. In ICALP, pages 901-912, 2007.

[7] Julien Cristau, Christof Löding, and Wolfgang Thomas. Deterministic automata on unranked trees. In FCT, pages 68-79, 2005.

[8] S. Demri and R. Lazić. LTL with the freeze quantifier and register automata. ACM Trans. Comput. Log., 10(3), 2009.

[9] D. Figueira. Satisfiability of downward XPath with data equality tests. In PODS, pages 197-206, 2009.

[10] D. Figueira. Forward-XPath and extended register automata on data-trees. In ICDT, pages 231-241, 2010.

[11] F. Geerts and W. Fan. Satisfiability of XPath queries with sibling axes. In DBPL, pages 122-137, 2005.

[12] M. Jurdziński and R. Lazić. Alternation-free modal mu-calculus for data trees. In LICS, pages 131-140, 2007.

[13] M. Kaminski and N. Francez. Finite-memory automata. Theor. Comput. Sci., 134(2):329-363, 1994.

[14] L. Segoufin. Automata and logics for words and trees over an infinite alphabet. In CSL, pages 41-57, 2006. 